\title{
LES ACIDES GRAS LIBRES DU FROMAGE
}

\author{
par
}

\author{
S. KUZDZAL-SAVOIE et W. KUZDZAL \\ Station centrale de Recherches laitières \\ et de Technologie des produits animaux \\ Jouy-en-Josas (Yvelines) France
}

Un fromage affiné contient toujours un certain taux d'acides gras libres (volatils et non volatils).

L'origine de ces acides est multiple : fermentations à partir du lactose, transformations des acides aminés résultant de la dégradation des protéines du lait, lipolyse.

Les acides gras libres contribuent à la formation de l'arôme du fromage. A chaque variété de fromage et aux meilleurs fromages de chaque variété, correspond sans nul doute un "profil " caractéristique des proportions relatives des divers acides gras libres. $\mathrm{Ce}$ profil "type " ou de "référence " ne peut être établi que si les quantités absolues des différents acides gras sont connues. Or, jusqu'à maintenant, ceci a été rarement obtenu.

En effet, la méthode de Harper (1953) permet d'obtenir un profil de l'ensemble des acides gras libres du fromage, mais les résultats sont exprimés en millimoles d'acides par rapport à un poids de fromage et seuls les acides volatils sont individualisés; seules les quantités absolues de ces acides volatils peuvent done être connues (Lehmann et Sahli, 1956). Si l'on attribue à l'ensemble des acides à plus de 6 atomes de carbone un poids moléculaire moyen (228 par exemple, chiffre choisi par Morris et al., 1955), il est possible d'exprimer les quantités des divers acides volatils libres en fonction des acides libres totaux, pour un poids donné de fromage.

De nombreux exemples d'application de la méthode de Harper à l'étude des fromages existent. Citons entre autres, les travaux de Harper (1959), de Willart (1960), de Kristoffersen et al. (1959, 1960), de Bachmann (1961).

La chromatographie en phase gazeuse s'est rapidement substituée à la chromatographie sur colonne pour l'étude des acides gras libres du fromage. Mais, à nouveau, les informations concernant les acides libres volatils (Ritter et Hanni, 1960 ; Sversen, 1961 ; Patton, 1963 et 1964 entre autres) furent beaucoup plus nombreuses que celles se rapportant à l'ensemble des acides libres (Bills et Day, 1964 ; Anderson et Day, 1965). 
Bills et Day (1964) ont publié une étude sur le fromage de Cheddar dans laquelle le "spectre" complet des acides libres (individualisation de tous les acides libres et détermination des quantités absolues de chacun d'entre eux) est présenté. A l'aide des mêmes techniques analytiques que celles utilisées par les auteurs précédents, Anderson et Day (1965) rapportèrent des informations comparables, relatives à plusieurs échantillons de fromages Bleus d'origine variée.

Ces auteurs ont appliqué une méthodologie complexe. En effet, les quantités d'acide acétique et d'acide butyrique sont déterminées séparément par deux méthodes différentes de chromatographie sur colonne : celle de Wiseman et Irvin (1957), pour l'acide acétique et celle de Keeney (1956) pour l'acide butyrique. Les quantités respectives des divers acides comptant plus de 4 atomes de carbone sont déterminées par chromatographie en phase gazeuse après transformation en esters méthyliques. Des étalons internes (acides heptanoïque et décaheptanoïque) sont utilisés en vue de l'analyse quantitative.

En fait, la méthode décrite s'applique bien au cas du 'Cheddar, puisque les acides gras libres ne renferment ni acide formique, ni acide propionique (Patton, 1963). Ces acides sont également absents selon Anderson et Day (1965) dans les acides libres des fromages Bleus.

Cependant Harper (1959) avait signalé la présence d'acide propionique dans un fromage Bleu, et Kristoffersen et al. (1960) ont également décelé cet acide dans divers échantillons de Cheddar ; enfin, selon Svensen (1961), on trouve dans les acides gras libres de divers fromages suédois de l'acide propionique, de l'acide isobu-, tyrique et de l'acide isovalérique. Ce dernier acide est également mis en évidence par Patton (1964a) dans le fromage suisse.

Or, en suivant strictement la méthode proposée par Bills et Day (1964) les acides volatils autres que l'acide acétique et l'acide butyrique, - surtout s'ils sont présents en faibles quantités sont difficilement dosables, alors que la chromatographie en phase gazeuse, grâce à sa quasi-universalité et à sa grande sensibilité, permet de déceler tous les acides volatils présents, même à l'état de traces.

La connaissance des quantités absolues de tous les acides gras libres présente un grand intérêt. En effet, si le taux des acides gras libres volatils, tels que les acides acétique, propionique et butyrique, est en rapport avec l'intensité des fermentations, le taux des acides libres à poids moléculaire moyen ou élevé est en rapport avec le degré de lipolyse et le taux des acides volatils ramifiés (isobutyrique, isovalérique) est en rapport avec l'activité des microorganismes protéolytiques. 
Compte tenu du rôle important que jouent les acides gras libres dans l'arôme du fromage, nous avons eherché à obtenir par une méthode aussi simple que possible, la totalité des acides gras libres du fromage en vue d'en effectuer ensuite l'analyse et le dosage spécifique au moyen de la chromatographie en phase gazeuse,

\section{I. - Méthode expérimentale}

\section{A) Les acides gras libres volatils.}

Pour le dosage des seuls acides libres volatils du fromage, la distillation a été utilisée. Nous avons adopté la technique décrite par Ritter et Hänni (1960) à la seule différence que le distillat était recueilli dans une solution aqueuse d'hydroxyde de sodium.

Après dessiccation des savons, les acides gras étaient libérés par hydrolyse selon la technique décrite par Roos et al. (1963) et directement injectés dans l'appareil de chromatographie en phase gazeuse.

\section{B) Les acides gras libres totaux.}

Pour le dosage des acides gras libres totaux, nous avons utilisé une méthode inspirée de la méthode proposée en 1958 par Hankinson et al., pour le dosage des acides gras libres volatils dans le lait.

Les acides gras libres sont extraits en milieu acide par l'alcooléther, puis neutralisés. Les savons sont séparés de la phase grasse et séchés. Une fraction est destinée au dosage des acides libres volatils. Une autre fraction est destinée au dosage des acides libres à poids moléculaire moyen et élevé.

L'utilisation de deux acides " étalons internes ", l'un à bas poids moléculaire, l'autre à poids moléculaire élevé, permet le dosage quantitatif de tous les acides libres.

Nous décrirons en détail la méthode proposée.

\section{1) Préparation des savons.}

$15 \mathrm{~g}$ environ de fromage sont broyés rapidement avec $20 \mathrm{ml}$ d'une solution aqueuse d'acide sulfurique à 10 p. $100(\mathrm{p} / \mathrm{p})$ et $50 \mathrm{ml}$ d'éthanol. Le tout est versé dans une ampoule à décantation. On ajoute $100 \mathrm{ml}$ d'oxyde d'éthyle et $100 \mathrm{ml}$ d'éther de pétrole (40-60). Après agitation et décantation, la solution éthérée est recueillie dans un Erlenmeyer. On ajoute à nouveau dans l'ampoule $50 \mathrm{ml}$ d'oxyde d'éthyle et $50 \mathrm{ml}$ d'éther de pétrole. Après agitation et décantation, la solution éthérée est jointe à la précédente fraction éthérée. Une troisième extraction peut être effectuée. 
Après addition de quelques gouttes d'une solution de phénolphtaléine dans l'éthanol (1 p. $100, \mathrm{p} / \mathrm{v}$ ), on titre rapidement la solution éthérée avec une solution aqueuse ou alcoolique d'hydroxyde de sodium $\mathrm{N} / 10$ ou $\mathrm{N} / 1$. On ajoute un excès d'hydroxyde de sodium.

Le tout est remis dans une ampoule à décantation et la solution de savons est séparée par décantation. Cette solution transvasée dans un bécher, est évaporée à sec, avec précaution, sur une plaque chauffante ou dans un évaporateur rotatif sous vide placé au bainmarie.

Les acides utilisés comme étalons internes peuvent être ajoutés au fromage ou dans la solution d'hydroxyde de sodium.

Les savons secs sont conservés à l'abri de la lumière et de l'humidité.

A ce stade les savons sont fractionnés en deux parties : la première partie est utilisée pour le dosage des acides volatils, la seconde pour le dosage des acides non volatils.

\section{2) Dosage des acides libres.}

Les acides volatils sont directement analysés par chromatographie en phase gazeuse après traitement des savons secs selon le procédé décrit par Roos et al. (1963). Les acides libres à plus haut poids moléculaire sont transformés en esters méthyliques. Les esters sont ensuite analysés par chromatographie en phase gazeuse.

Le dosage des acides volatils libres obtenus par la méthode d'extraction peut être effectué indépendamment du dosage des acides libres à plus haut poids moléculaire. C'est ce qui a été effectué dans la présente étude.

\section{C) Chromatographie en phase gazeuse.}

Nous avons utilisé un appareil Aerograph Hy Fi 600 à détecteur à ionisation de flamme.

Pour l'analyse directe des acides gras nous avons utilisé une colonne en acier inoxydable de $1,5 \mathrm{~m}$ environ ( 5 pieds) de long et de $0,3 \mathrm{~cm}$ environ de diamètre interne ( $1 / 8^{\mathrm{e}}$ de pouce) chargée à 10 p. 100 de succinate de diéthylène glycol (DEGS) contenant 2 p. 100 d'acide phosphorique, sur brique à feu 80/100 mesh. La température de la colonne était de $120^{\circ} \mathrm{C}$ environ, et les débits d'hydrogène et d'azote (gaz vecteur) de $30 \mathrm{ml} /$ minute environ.

Pour l'analyse des esters méthyliques nous avons opéré dans les conditions habituelles. 


\section{D) Détermination du degré de lipolyse.}

Celui-ci est déterminé sur la matière grasse du fromage obtenue après extraction du fromage, mélangé au double de son poids de sable et à son poids de sulfate de soude anhydre, par l'éther de pétrole $\left(40-60^{\circ}\right)$ dans un appareil (BBS) d'extraction continue (16 heures).

La détermination est effectuée selon la technique décrite dans la norme FIL no 9 .

\section{II. - Résultats}

\section{1) Comparaison des résultats obtenus par la méthode d'extraction proposée et la méthode de distillation (acides volatils seulement).}

Sur une série d'échantillons de fromages de Gruyère de Comté on a procédé aux analyses des acides volatils selon la méthode de distillation et selon la méthode d'extraction. Dans cette série d'analyses, on n'a pas utilisé d'étalon interne.

On a done obtenu une série de chromatogrammes sur lesquels sont visibles les acides de $\mathrm{C}_{2}$ à $\mathrm{C}_{6}$. Dans les fromages de Gruyère de Comté, ce dernier acide est présent en très faible quantité par rapport aux acides à 2,3 et 4 atomes de carbone. On a calculé les proportions relatives des acides acétique $\left(\mathrm{C}_{2}\right)$, propionique $\left(\mathrm{C}_{3}\right)$ et butyrique $\left(\mathrm{C}_{4}\right)$ dans les divers échantillons, à partir des chromatogrammes obtenus.

Les résultats sont portés dans le tableau I. A titre d'exemple, la figure 1 donne une image des chromatogrammes obtenus soit par la méthode de distillation, soit par la méthode d'extraction à partir de deux échantillons de fromage.

On constate que la méthode par extraction permet d'obtenir une proportion plus forte d'acide acétique que la méthode par distillation, par contre le rapport acide butyrique/acide propionique est assez voisin selon que l'une ou l'autre méthode est utilisée. Il semble donc que la méthode par extraction réduise sensiblement les pertes en acide acétique.

\section{2) Etude des «profils « d'acides volatils des Gruyères de Comté.}

a) Sans étalon interne.

Dans tous les Gruyères de Comté analysés (tableau I), l'acide propionique est l'acide volatil majeur; il représente 60 à 80 p. 100 des acides de $\mathrm{C}_{2}$ à $\mathrm{C}_{4}$ inclus. Cependant, le profil présenté sans addition d'étalon interne ne permet pas de caractériser la fermentation 
ÉTUDE DES ACIDES GRAS VOLATILS LIBRES, DANS DIVERS ÉCHANTILLONS DE "GRUY k̀RE DE COMTÉ " COMPARAISON DES MÉTHODES : DISTILLATION EXTRACTION

(Résultats exprimés en p. 100 des acides volatils de $\mathrm{C}_{2}$ à $\mathbf{C}_{5}$ )

\begin{tabular}{|c|c|c|c|c|c|c|c|c|c|c|c|c|c|c|}
\hline \multirow{3}{*}{$\begin{array}{c}\text { Désignation } \\
\text { des acides } \\
\text { et rapports }\end{array}$} & \multicolumn{8}{|c|}{$\begin{array}{l}\text { Fromages fabriqués en mai } 1965 \text {, } \\
\text { ouverts le } 22 \text { novembre } 1965\end{array}$} & \multicolumn{6}{|c|}{$\begin{array}{c}\text { Fromages fabriqués en juillet } 1965 \text {, } \\
\text { ouverts le } 22 \text { novembre } 1965\end{array}$} \\
\hline & $\mathbf{T}_{10}$ & $\begin{array}{l}51,6 * \\
3,2 * *\end{array}$ & $\mathrm{E}_{10}$ & $\begin{array}{l}48,7 * \\
7,6 * *\end{array}$ & $\mathbf{E}_{7}$ & $\begin{array}{l}45,6^{*} \\
6,6^{* *}\end{array}$ & $\mathbf{E}_{9}$ & $\begin{array}{l}47,3 * \\
3,8 * *\end{array}$ & $\mathrm{~T}_{3}$ & $\begin{array}{l}15,9 * \\
3,5 * *\end{array}$ & $\mathrm{E}_{3}$ & $\begin{array}{l}46,4^{*} \\
3,8 * *\end{array}$ & $\mathrm{~T}_{7}$ & $\begin{array}{l}48,6^{*} \\
4,6 * *\end{array}$ \\
\hline & Dist. & Extr. & Dist. & Extr. & Dist. & Extr. & Dist. & Extr. & Dist. & Extr. & Dist. & Extr. & Dist. & Extr. \\
\hline Acide acétique $\left(\mathrm{C}_{2}\right) \ldots \ldots$ & 11,1 & 12,9 & 9,9 & 13,3 & 8,3 & 15,1 & 7,9 & 14,1 & 10,3 & 17,4 & 9,3 & 15,9 & 14,2 & 19,3 \\
\hline Acide propionique $\left(\mathrm{C}_{3}\right) \ldots$ & 76,3 & 76,2 & 74,1 & 73,6 & 60,7 & 55,8 & 64,5 & 60,6 & 77,0 & 69,4 & 84,1 & 75,2 & 80,8 & 72,5 \\
\hline Acide butyrique $\left(\mathrm{C}_{4}\right) \ldots \ldots$ & 12,0 & 10,4 & 15,2 & 12,5 & 30,0 & 28,6 & 26,8 & 24,3 & 10,5 & 11,2 & 6,0 & 7,3 & 3,8 & 4,8 \\
\hline Isovalérique (?) $\left(\mathrm{C}_{5}\right) \ldots \ldots$ & 0,6 & 0,5 & 0,8 & 0,6 & 0,5 & 0,5 & 0,8 & 1,0 & 2,2 & 2,0 & 0,6 & 1,6 & 1,2 & 2,4 \\
\hline $\mathrm{C}_{2} / \mathrm{C}_{3}$ p. $100 \ldots \ldots \ldots \ldots$ & 14,7 & 16,9 & 13,4 & 18,1 & 13,7 & 27,1 & 12,3 & 24,5 & 13,4 & 25,1 & 11,2 & 21,3 & 17,6 & 26,8 \\
\hline $\mathrm{C}_{4} / \mathrm{C}_{3}$ p. $100 \ldots \ldots \ldots \ldots$ & 16,6 & 14,3 & 21,6 & 17,9 & 51,1 & 52,3 & 42,8 & 41,2 & 13,8 & 16,2 & 7,2 & 9,7 & 5,2 & 6,7 \\
\hline
\end{tabular}

* Gras/sec.

** Acidité libre (en ml NaOH N/1 p. 100 g mat. gr.). 

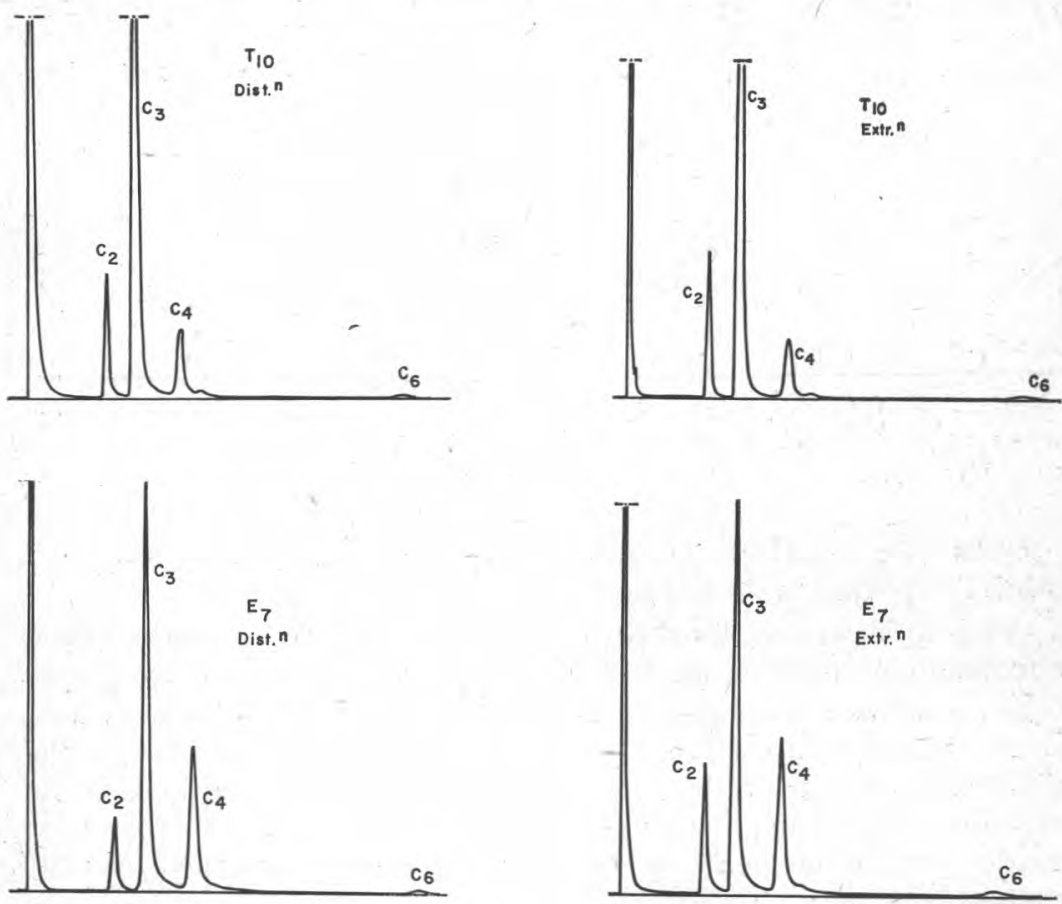

Fig. 1. - Analyse par chromatographie en phase gazeuse des acides volatils libres du Gruyère de Comté. - Comparaison de la méthode de distillation et de la méthode d'extraction :

en haut : fromage $\mathrm{T}_{10}$; en bas: fromage $\mathrm{E}_{7}$.

Chaque pic représente un acide gras désigné par son nombre d'atomes de carbone.

$$
\begin{array}{ll}
\mathrm{C}_{2}=\text { acide acétique. } & \mathrm{C}_{3}=\text { acide propionique. } \\
\mathrm{C}_{4}=\text { acide butyrique. } & \mathrm{C}_{6}=\text { acide caproïque. }
\end{array}
$$

propionique, de préciser si elle est faible ou si elle est intense. Pour cela, il est nécessaire de connaître le taux des acides volatils en fonction d'un poids de fromage. Cependant l'établissement du profil des acides volatils permet de connaître la proportion entre les acides propionique et butyrique : elle varie beaucoup d'un fromage à l'autre.

Dans un fromage de Comté de trois mois et demi, présentant le défaut de gonflement butyrique, nous avons obtenu le profil présenté sur la figure 2 (méthode de distillation). L'acide butyrique est dans ce cas, l'acide volatil majeur et représente près de trois fois la quantité d'acide propionique. 

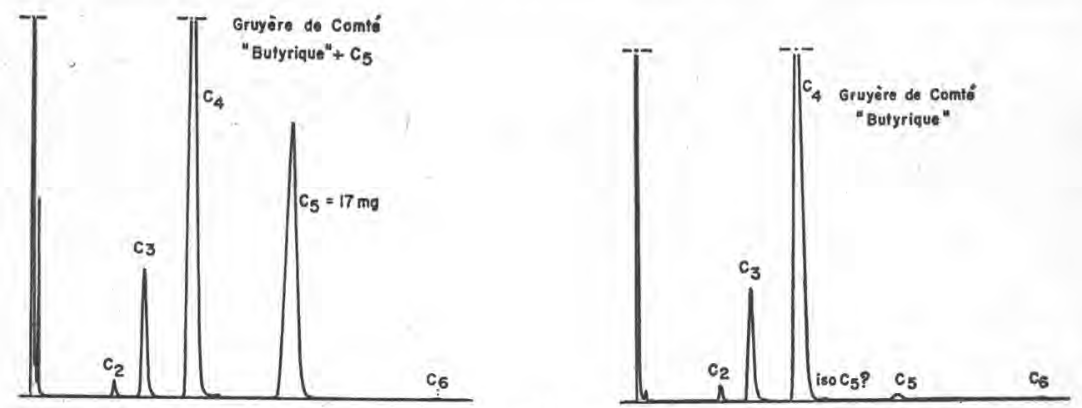

Fig. 2. - Analyse par chromatographie en phase gazeuse des acides volatils libres d'un Gruyère de Comté "butyrique ", avee et sans addition d'étalon interne $\left(\mathrm{C}_{5}=\right.$ acide valérique $)$.

Dans la plupart des échantillons de fromages analysés on a évalué l'importance de la lipolyse.

Cette lipolyse est donnée par l'acidité libre de la phase grasse du fromage, évaluée en $\mathrm{ml} \mathrm{NaOH} \mathrm{N/1}$ pour $100 \mathrm{~g}$ de matière grasse.

Les résultats concernant la première série d'échantillons sont portés dans le tableau 1. Une lipolyse caractérisée modifie peu les proportions relatives des trois acides acétique, propionique et butyrique. Tout au plus serait-on tenté de dire que la proportion d'acide butyrique peut se trouver légèrement accrue (cas du fromage $\mathrm{E}_{7}$ ).

L'acide butyrique libre pouvant avoir diverses origines, ne peut être utilisé pour caractériser la lipolyse. L'acide caproïque libre (hexanoïque) convient certes mieux puisque son origine est purement lipolytique. Cet acide est visible sur les chromatogrammes obtenus; cependant, son taux étant très faible par rapport aux autres acides volatils, une variation de ce taux n'est pas très facile à mettre en évidence. De plus, dans la méthode utilisée, sans étalon interne, on ignore les quantités absolues des acides volatils par rapport au poids de fromage, si bien que, par exemple, une forte lipolyse jointe à une fermentation propionique intense (dans la mesure où la chose existe) pourrait conduire à un "profil " d'acides volatils (de $\mathrm{C}_{2}$ à $\mathrm{C}_{6}$ ) semblable au profil obtenu dans un cas de faible lipolyse accompagnant une faible fermentation propionique.

Ainsi l'intérêt de connaître les quantités absolues des différents acides libres du fromage est une fois de plus démontré.

b) Avec addition d'étalon interne.

Nous avons done effectué une nouvelle série d'analyses sur d'autres échantillons de Gruyère de Comté en vue d'obtenir ces quantités absolues. Ceci nécessite l'emploi d'un étalon interne. Nous avons choisi l'acide valérique $\left(\mathrm{C}_{5}\right)$ et les résultats analytiques obtenus ont été rassemblés dans le tableau II. 
TABLEAU II

ETUDE DES ACIDES GRAS VOLATILS DANS DIVERS GOHANTILLONS DE "GRUYłiRE DE COMTÉ " (Résultats pondéraux)

\begin{tabular}{|c|c|c|c|c|c|c|c|c|c|c|c|c|}
\hline \multirow{2}{*}{ Echantillon } & \multirow{2}{*}{$\begin{array}{c}\text { Date } \\
\text { de fabrication }\end{array}$} & \multirow{2}{*}{$\begin{array}{c}\text { Date } \\
\text { d'ouverture }\end{array}$} & \multirow{2}{*}{$\begin{array}{c}\text { Date } \\
\text { d'analyse }\end{array}$} & \multirow{2}{*}{$\begin{array}{l}\text { Grasl } \\
\text { sec }\end{array}$} & \multirow{2}{*}{$\begin{array}{c}\text { Acidité } \\
\text { libre }\end{array}$} & \multicolumn{7}{|c|}{$\begin{array}{c}\text { Acides gras } \\
\text { en } m g \text { p. } 100 \mathrm{~g} \text { de fromage }\end{array}$} \\
\hline & & & & & & $\mathbf{C}_{2}$ & $\mathrm{C}_{3}$ & $\mathrm{C}_{4}$ & $i s o \mathrm{C}_{5}$ & $\mathrm{C}_{5}$ & iso $\mathrm{C}_{6}$ & $\mathbf{C}_{6}$ \\
\hline $\mathbf{T}_{3} \ldots \ldots \cdots$ & Octobre 65 & $30-3-66$ & $20-4-66$ & 45,3 & 3,1 & 31 & 250 & 30 & 10 & 3 & 11 & 6 \\
\hline $\mathrm{E}_{2} \ldots \ldots \ldots \ldots$ & Octobre 65 & 30-3-66 & 28-4-66 & 48,2 & 6,2 & 50 & 301 & 71 & 19 & 4 & 21 & 14 \\
\hline $\mathbf{T}_{2}$ (centre) $\ldots$ & Octobre 65 & 30-3-66 & 26-4-66 & 47,2 & 3,8 & 20 & 160 & 35 & 9 & 2 & 4 & 6 \\
\hline $\mathbf{T}_{2}$ (périphérie) .. & Octobre 65 & $30-3-66$ & $26-4-66$ & 47,5 & $\mathbf{3 , 8}$ & 27 & 183 & 74 & 9 & 6 & 4 & 6 \\
\hline $\mathrm{V}^{* *} \ldots \ldots \ldots$ & Décembre 65 & $3-4-66$ & $6-5-66$ & - & - & 2 & 13 & 71 & 1 & 2 & - & 1 \\
\hline
\end{tabular}

* Exprimée en $\mathrm{ml}$ de $\mathrm{NaOH} \mathrm{N} / 1$ pour $100 \mathrm{~g}$ de matière grasse.

** Gonflement butyrique. 
Des reproductions de quelques chromatogrammes obtenus sont présentés sur la figure 3 .

On voit ainsi que dans un fromage où la lipolyse est relativement prononcée (le $\mathrm{E}_{2}$ de mars 1966 par exemple), le taux d'acide caproïque $\left(\mathrm{C}_{6}\right)$ libre pour un poids donné de fromage est relativement fort.
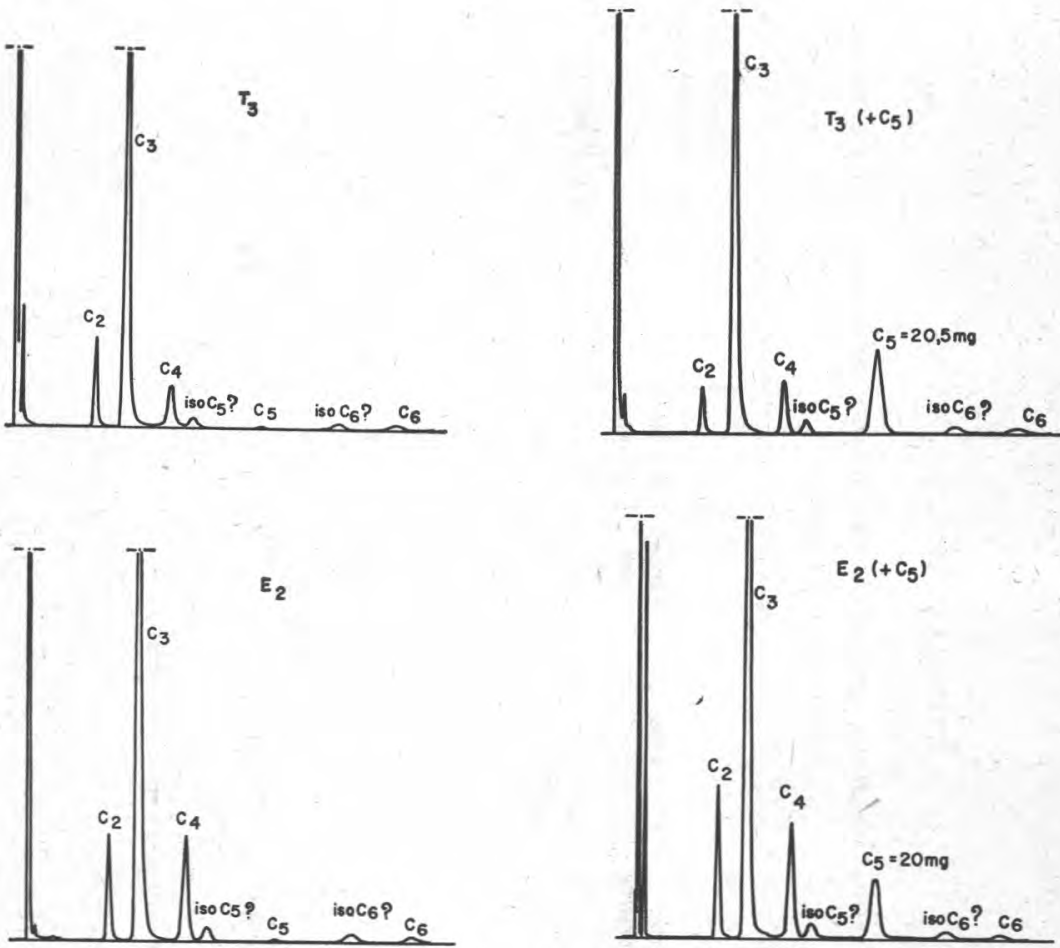

Fig. 3. - Analyse par chromatographie en phase gazeuse des acides volatils libres de deux Gruyères de Comté, avec et sans addition d'étalon interne $\left(\mathrm{C}_{5}\right)$ : en haut : fromage $\mathrm{T}_{\mathbf{3}}$. en bas : fromage $\mathrm{E}_{\mathbf{2}}$.

Dans l'échantillon de fromage présentant un gonflement butyrique (tableau II, fig. 2), le taux de l'acide propionique est relativement faible.

Nous avons également étudié les acides volatils de deux échantillons de fromage Emmental, l'un normal et l'autre présentant un gonflement butyrique. Les résultats sont portés dans le tableau III.

Les quantités absolues d'acides libres sont plus faibles dans les Emmental que dans les Gruyères de Comté, ce qui peut être dû au fait que les Emmental sont plus jeunes que les Gruyères de Comté. 
TABLEAU III

ÉtUde DES ACIDES GRAS volatils LIBRes DANS DIVERS ÉCHANTILLONS D'EMMENTAL

(Résultats pondéraux)

\begin{tabular}{|c|c|c|c|c|c|c|c|c|c|c|c|}
\hline \multirow{2}{*}{. } & \multirow{2}{*}{$\begin{array}{c}\text { Date } \\
\text { de fabrication }\end{array}$} & \multirow{2}{*}{$\begin{array}{c}\text { Date } \\
\text { d'ouverture }\end{array}$} & \multirow{2}{*}{$\begin{array}{l}\text { Date } \\
\text { d'analyse }\end{array}$} & \multirow{2}{*}{$\begin{array}{l}\text { gras/ } \\
\text { sec }\end{array}$} & \multirow{2}{*}{$\begin{array}{l}\text { Acidité } \\
\text { libre* }\end{array}$} & \multicolumn{6}{|c|}{ Acides gras en mg p. $100 \mathrm{~g}$ de fromage } \\
\hline & & & & & & $\mathrm{C}_{2}$ & $\mathrm{C}_{3}$ & $\mathrm{C}_{4}$ & iso $\mathrm{C}_{5}$ & $\mathrm{C}_{5}$ & $\mathrm{C}_{6}$ \\
\hline $\begin{array}{l}* * \text { Emmental } \\
\text { 101-centre } \ldots \ldots \ldots\end{array}$ & $24-1-66$ & 22-3-66 & 31-3-66 & 46,5 & 2,2 & 7 & 12 & 27 & 2 & 1 & 2 \\
\hline $\begin{array}{l}\text { **Emmental } \\
\text { 101-périphérie ... }\end{array}$ & 24-1-66 & $22-3-66$ & $1-4-66$ & & & 12 & 40 & 62 & 3 & 3 & 4 \\
\hline $\begin{array}{l}* * * \text { Emmental } \\
\quad 127-\ldots \ldots \ldots \ldots\end{array}$ & $17-2-66$ & $26-4-66$ & $6-5-66$ & & & 23 & 180 & 21 & 5 & 6 & 8 \\
\hline
\end{tabular}

* En $\mathrm{ml} \mathrm{NaOH} \mathrm{N/1}$ pour $100 \mathrm{~g}$ de matière grasse.

** Fromage jeune, peu ouvert au centre, fort gonflement butyrique à la périphérie (présence de Clostridia à l'analyse bactériologique).

*** Fromage normal. 


\section{III. - Discussion}

Nous avons proposé une méthode permettant d'obtenir en une seule opération tous les acides gras libres du fromage.

De nombreuses informations avaient déjà été acquises en ce qui concerne les acides volatils libres grâce à la méthode de Harper (1959). Cependant la chromatographie en phase gazeuse est plus simple, plus rapide, beaucoup plus sensible et permet d'obtenir une bonne résolution des acides intermédiaires.

La précision varie avec la reproductibilité des chromatogrammes. Les conditions opératoires doivent être bien définies et fidèlement respectées pour obtenir le maximum de reproductibilité.

Des acides gras libres présents à l'état de traces peuvent être décelés. Ces acides volatils mineurs peuvent contribuer à nuancer l'arôme d'un fromage. Ainsi par exemple, nous avons nettement décelé dans des fromages de Gruyère de Comté fabriqués avec du lait d'automne (mois d'octobre) et seulement dans ceux-là, un acide gras dont le temps de rétention est un peu plus court que celui de l'acide hexanoïque (ou acide caproïque).

Nous avons toujours trouvé une quantité relativement faible d'acide acétique. Une légère perte n'est pas exclue au cours de la dessiccation des sels de sodium (Hankinsol et al., 1958). Cependant Patton (1964a) a présenté un profil d'acides volatils de «Swiss cheese " tout à fait semblable à ceux que nous avons nous-mêmes présentés au cours de cette étude. Un profil de "Swiss cheese " présenté par Svensen (1961) montre par contre une plus forte proportion d'acide acétique atteignant environ 50 p. 100 de l'acide propionique. En 1956, Lehmann et Sahli ont signalé dans le fromage Emmental de très fortes quantités d'acide acétique pratiquement équivalentes aux quantités d'acide propionique. En se basant sur l'étude de Harper (1959), il est permis de penser que l'acide pyruvique pouvait interférer. Cette interférence, possible dans le cas de la chromatographie sur colonne, ne peut se produire dans le cas de la chromatographie en phase gazeuse, compte tenu du point de volatilisation élevé de l'acide pyruvique.

Il reste cependant que les plus grandes précautions doivent être prises pour éviter toute perte d'acide acétique. Le taux de cet acide est en effet très utile à connaître car il constitue dans certains fromages affinés, tels le Cheddar, l'acide volatil majeur. Il est aussi toujours important au début de l'affinage et les variations du taux de cet acide peuvent apporter d'utiles indications.

Les exemples choisis au cours de cette étude n'ont eu pour but que de montrer les possibilités d'investigations offertes par la méthode proposée. 
La gamme étendue des fromages français se prête en effet à l'exploitation de cette méthode. La méthode de distillation peut être utilisée avec profit pour le dosage des acides gras libres dans le cas du Gruyère de Comté ou de l'Emmental puisque les acides acétique, propionique et butyrique représentent ensemble, plus de 50 p. 100 des acides libres totaux. Cependant, l'emploi de la méthode par extraction, que nous avons proposée, réduirait sensiblement les pertes en acide acétique.

Dans les fromages Bleus (Anderson et Day, 1965), les acidess à 2 et 4 atomes de carbone ne représentent qu'environ 5 p. 100 des acides libres totaux; de même, dans le cas des fromages à pâte molle.

Pour l'étude de ces deux groupes de fromage, la méthode d'extraction permet d'obtenir le profil complet des acides libres et semble mieux indiquée, puisque les acides à chaîne moyenne ou longue constituent la fraction majeure des acides libres.

Récemment, Scanlan et al. (1965) ont montré que les acides libres de 4 à 12 atomes de carbone (nombre pair) contribuent chacun également à la formation du goût de "rance ". Ceci s'accorde assez bien avec les résultats de Patton $(1964 b)$ qui trouve pour ces acides, en solution aqueuse, pratiquement un seuil uniforme de perception, alors qu'en solution huileuse le seuil de perception des acides gras s'élève en même temps que la chaîne s'allonge (Féron et Govignon, 1961).

Il convient aussi de rappeler que, dans le fromage, les sels des acides gras (savons) peuvent introduire un élément spécifique d'arôme (Anderson et Day, 1965).

Par ailleurs, l'origine de l'acide acétique et son rôle dans l'arôme du fromage sont encore assez mal établis (Patton, 1963).

Ainsi les techniques analytiques décrites et utilisées dans la présente étude ouvrent la voie qui devrait rapidement conduire à l'établissement de corrélations entre l'analyse des acides gras libres et les résultats de l'examen organoleptique ou celui de l'analyse bactériologique, permettant ainsi une meilleure compréhension des phénomènes biochimiques de l'affinage.

Nous remercions MM. J. Auclair et J. L. Bergère, qui nous ont procuré les échantillons de fromage analysés dans cette étude.

\section{Résumé}

Une méthode permettant l'obtention de l'ensemble des acides gras libres du fromage est décrite. La chromatographie en phase gazeuse (avec addition d'un étalon interne) permet le dosage quantitatif des divers acides gras. 
Des exemples d'application à l'étude des acides gras libres de quelques Gruyère de Comté illustrent les possibilités d'investigations offertes par la méthode proposée.

\section{Summary}

\section{The free fatty acids of cheese.}

The origin and the role of free fatty acids in matured cheese have been discussed.

The level of the free fatty acids (per weight of cheese) and the relative proportions of the different free fatty acids constitute specific characteristics according to the different types of cheese.

The authors propose a rather easy method by means of which it is possible to obtain the "complete spectrum" of free fatty acids present in a cheese sample.

The free fatty acids are obtained by extraction from acidified cheese and then transformed into soaps. After desiccation of the soaps, the fatty acids are freed by acid hydrolysis. The volatile fatty acids are analysed directly by gas chromatography and the fatty acids with a higher molecular weight are also analysed by gas chromatography, but only after transformation into methyl esters.

The quantitative determination of all free acids is possible when using two acids, "internal standards", the one having a low molecular weight and the other a high molecular weight.

The authors show that by using the proposed extraction method one obtains a greater quantity of acetic acid than by the distillation method, however, it is not possible to state that the whole quantity of acetic acid has been determined.

The proposed method has been applied to the study of free fatty acids in different samples of the following cheeses : "Gruyère de Comté" and "Emmental" which are very different as far as the organoleptic quality is concerned.

Manuscrit reçu en octobre 1966.

\section{RÉFÉRENCES BIBLIOGRAPHIQUES}

Anderson (D. F.), Day (E. A.). J. Dairy Sci., 1965, 48, 248.

BAOHMANN (M.). Promotion arbeit (no 3043). Eidgenössischen Technischen Hochschule, Zürich.

Bills (D. D.), DAy (E. A.). J. Dairy Sci., 1964, 47, 733.

F́ron (R.) et Govignon (M.). Ann. Fals. Expert, Chim., 1961, 54, 308. 
Hankinson (C. L.), Harper (W. J.) et Mrkolatoik (E.). J. Dairy Sci., $1958,41,1502$.

HARPER (W. J.). J. Dairy Sci., 1953, 36, 808.

Harper (W. J.). J. Dairy Sci., 1959, 42, 207.

Keeney (M.). J. Assoc. Ofic. Agric. Chemists, 1956, 39, 212.

Kristoffersen (T.), Gould (I. A.), Harper (W. J.). Milk Prod. J., 1959, $50,14$.

Kristoffersen (T.). Gould (I. A.), J. Dairy Sci., 1960, 43, 1202.

Lehmann (W. von), SAHLI (K.). XIVe Congrès intern. Lait, Rome, 1956, 2,315 .

Morris (H. A.), Jezeski (J. J.), Сombs (W. B.). Kuramoto (S.), J. Dairy Sci., $1963,46,1$.

Patton (S.). J. Dairy Sci., 1963, 46, 856.

Patton (S.). J. Dairy Sci., 1964a, 47, 817.

Patton (S.). Food Sci., 1964b, 29, 679.

Ritter (W.). Hänni (H.), Milchwissens., 1960, 15, 296.

Roos (J. B.). Versnel (A.) et Werdmuller (G. A.), Kieler Milchwirts, Forch, 1963, 15, 515 .

Scanlan (R. A.). Sather (L. A.) et Day (E. A.), J. Dairy Sci., 1965, 48, 1582.

Svensen (A.). Rapport no 97, Dairy Res. Institute, Oslo, 1961 (Meierisposten $\mathrm{nr}$. 12-13-14).

Willart (S.). XIVe Cong. intern. Lait, Rome, 1956, 3, 711.

Wiseman (A. G.) et Irvin (H. M.). J. Agric. Food Chem., 1957, 5, 213. 